\title{
Does enteral nutrition infusion rate impact on the gastric residual volume of critically III Patients?
}

\begin{abstract}
In critically ill patients, enteral nutrition is considered the main method of nutritional support. However, gastrointestinal complications pose a challenge to an appropriate nutritional supply. Gastric residual volume (GRV) is routinely measured in critical care patients. The aim of this work was to evaluate the GRV measurements of in critically ill patients and its linking with an increase in the infusion rate (IR) of enteral nutrition. The study was conducted with 25 male and female patients, older than 18years old, who were hospitalized in the Intensive Care Unit (ICU) with exclusive enteral nutrition for at least 72hours. The GRVs were collected from records of the Clinical Nutrition Unit, as well as the increase in IR and nutritional adequacy. The mean age for the sample was $63 \pm 15$ years, with a higher prevalence for male sex $(56 \%)$. The GRV, before and after the increase in IR, presented the medians 10 and 23 , respectively. Most patients $(52 \%)$ did not reach $80 \%$ of the protein-calorie recommendations within 72 hours from the beginning of the enteral nutrition. We found a significant negative correlation $(r=-0.46 ; \mathrm{p}=0.02)$ between the increase in IR and GRV. The results indicate that the increase of the enteral nutrition infusion rate did not positively affect the GRV of critically ill patients. Other factors are likely more determinant for gastric emptying and assessment of enteral nutrition tolerance.
\end{abstract}

Volume II Issue 2 - 2020

\author{
Poliana Guiomar de Almeida Brasiel,' \\ Adriana Soares Torres Melo,' Aline Silva \\ de Aguiar,2 Sheila Cristina Potente Dutra \\ Luquetti ${ }^{2}$ \\ 'Clinical Nutrition Unit, University Hospital - Federal University \\ of Juiz de Fora, Brazil \\ ${ }^{2}$ Department of Nutrition, Federal University of Juiz de Fora, \\ Brazil
}

Correspondence: Poliana Guiomar A, Brasiel, University Hospital of the Federal University of Juiz de Fora, Street Catulo Breviglieri, $s / n^{\circ}$. Santa Catarina, 36036 I I0, Juiz de Fora, Brasil, Email guidinhabra@hotmail.com

Received: February II, 2020 | Published: April 14, 2020

Keywords: gastric motility, gastric residual volume, intensive care unit, enteral nutrition

Abbreviations: GRV, gastric residual volume; ICU, intensive care unit; IR, infusion rate; EN, enteral nutrition; $\mathrm{mL}$, milliliters

\section{Introduction}

Enteral nutrition $(\mathrm{EN})$ is the main route of feeding for patients in the Intensive Care Unit (ICU). Adequate nutritional support is associated with a reduction in morbidity and mortality rates and with the improvement of the clinical outcome of these patients. However, studies indicate that an early nutritional therapy often does not occur and there is often a failure to supply adequate calories and proteins to meet the patient's needs; Gastrointestinal intolerance to EN can be a difficulty attributed to this inadequacy. Gastroparesis causing increased gastric residual volume, gastroesophageal reflux, abdominal pain and distension, diarrhea, vomiting, risk of bronchoaspiration and ventilator-associated pneumonia are common complications to critically ill patients. ${ }^{1-4}$

All of these factors contributed to the routine use of the gastric residual volume (GRV) when monitoring patients on enteral nutritional therapy. The GRV is a value of direct measurement of stomach contents, ascertained by aspiration through the enteric tube. The limit value is defined for the tolerance of the EN. When volumes exceed this value, nutrition is suspended or delayed. ${ }^{5,6}$ However, there is no consensus on the limit value to be used, and there is a limited number of studies that have evaluated the use and validity of this indicator of gastric tolerance. ${ }^{1,5}$

There is extensive discussion currently about the use and definition of tolerable GRV for maintenance of EN therapy. In 2016, the American Society for Parenteral and Enteral Nutrition (ASPEN) and the Society of Critical Care Medicine (SCCM) proposed not to routinely use the GRV in the monitoring of ICU patients receiving
EN. ${ }^{7}$ Current recommendations, which guide the international guidelines for parenteral and enteral nutrition, have raised this value over theyears. As well as reinforcing the importance of implementing measures that include post-pyloric continuous nutrition and the use of prokinetics, which should be considered when maintaining high GRV (greater than 500mL) ${ }^{8,9}$ The misuse of this marker appears to be associated with an increased risk of nutrition tube obstruction, inappropriate health resource allocation, and inadequate nutrition suspension, with a reduction in nutritional supply. ${ }^{7}$

The relationship between the increase in GRV and the occurrence of regurgitation, bronchoaspiration and pneumonia is controversial. Thus, the validity of the evaluation of the GRV as a good indicator of EN tolerance and associated complications is questioned..$^{10}$ The present study aims to evaluate the association of increased infusion rate of the EN and the GRV routinely monitored in critically ill patients.

\section{Patients and methods}

After approval by the Committee of Ethics in Research on Human Beings of the University Hospital of the Federal University of Juiz de Fora (No. 2.458.173), Minas Gerais, Brazil, the data were collected from protocols routinely registered by the Clinical Nutrition Unit / Nutrition Therapy of the institution. Twenty-five critical patients admitted to the University Hospital of the Federal University of Juiz de Fora (HU-UFJF/Ebserh) and accompanied by the nutrition service were included in this retrospective study. Patients older than 18years old, who remained in EN for an exclusive enteral tube feeding for at least 72hours, and presented complete protocol in recording, were considered eligible to participate in the study. Patients who presented jejunostomy, enteral tube in post-pyloric positioning, or remained in ICU and EN for less than 7hours were considered ineligible. 
Data including sex and age, medical admission diagnosis, Body Mass Index (BMI) and occurrence or non-occurrence of pulmonary aspiration were collected from the patients' records. Values of caloric and protein adequacy were collected from service protocols.

Considering the protocol applied by the Nutrition Therapy Service to reach the goal of at least $80 \%$ of the caloric and protein recommendations within 3 days of EN initiation and the gastrointestinal complications presented by the patients, the infusion rate of the EN and its evolution over the days were individually determined by the responsible nutritionists. The measurements of GRV values were performed every 12 hours ( 2 times a day), considering $350 \mathrm{~mL}$ as the maximum tolerable value for maintenance of $\mathrm{EN}$, following the protocol applied. When higher values $(>350 \mathrm{ml})$ were found, the EN was suspended. It should be noted that the positioning of the nasogastric tube was confirmed using a chest X-ray. Patients received a commercial enteral formula in a closed system, fiber free, according to a specific recommended rate via a continuous pump feeding. A weight-based predictive equation was used to guide caloric and protein prescription. During the monitoring period, the head of the bed was kept elevated (to $30-45^{\circ}$ ).

The GRV was measured in milliliters $(\mathrm{mL})$, aspirating the gastric contents with a $20 \mathrm{~mL}$ syringe; if a volume of less than $350 \mathrm{~mL}$ was obtained from the patient, it was returned.

\section{Statistical analysis}

A statistical analysis was performed using the statistical software SPSS version 23.0 (IBM SPSS Statistics, Armonk, NY, USA). To characterize the sample, a descriptive analysis was carried out with

Table I General characteristics of the study population frequency, central tendency and dispersion measurements. After the analysis of the data normality, (Shapiro-Wilk test), comparisons of the continuous variables were conducted by the non-parametric Mann-Whitney test. The correlation evaluations were verified by the Spearman's correlation, being considered as statistically significant when $\mathrm{p}<0.05$.

\section{Results}

From a total of 25 individuals, 14 (56\%) were male. The mean age of the patients was $63.7 \pm 15$ years, with the majority $(60 \%)$ being evaluated in the age group of 18 to 65 years. The most frequent medical specialties were hematology $(24 \%)$, surgery $(16 \%)$, gastroenterology $(16 \%)$ and infectology $(12 \%)$. According to the BMI classification for the age group, $9(36 \%)$ of the patients were overweight or obese, 9 $(36 \%)$ were normal weight and $7(28 \%)$ were underweight. Table 1 presents the general characteristics of the population evaluated.

A median of 10 (IQR 0- 200mL) was found to assess the GRV prior to an increased infusion rate of the diet. After increasing the rate of infusion, the volume of 23 (IQR $0-210 \mathrm{~mL}$ ) was observed. There was no significant difference between the GRV before and after the infusion rise of EN.

For indicators of nutritional adequacy, the goal of the service is to reach at least $80 \%$ of the caloric and protein recommendations within 72 hours (3days). In this way, $12(48 \%)$ of the patients evaluated, reached this value. Regarding the variation in the GRV after the increase of the infusion rate of the diet, 15 individuals $(60 \%)$ presented increased GRV, 7 (28\%) reduction and $3(12 \%)$ maintenance (Table 2).

\begin{tabular}{llll}
\hline Characteristics & & N & $\%$ \\
\hline \multirow{2}{*}{ AGE } & I8 to 65years & 15 & 60 \\
& 65years or more & 10 & 40 \\
SEX & Male & 14 & 56 \\
& Female & 11 & 44 \\
& Hematology & 6 & 24 \\
Diagnosis Category & Gastroenterology & 4 & 16 \\
& Surgery & 4 & 16 \\
& Infectology & 3 & 12 \\
& Other & 8 & 32 \\
Nutritional Status (BMI) & Underweight & 7 & \\
& Normal weight & 9 & 28 \\
& Overweight or Obesity & 9 & 36 \\
\end{tabular}

Table 2 Monitoring nutritional adequacy and volume of gastric residue

\begin{tabular}{llll}
\hline Monitoring & N & $\%$ \\
\hline Adequacy of Calorie Intake & $\geq 80 \%$ within $72 \mathrm{~h}$ & 12 & 48 \\
& $<80 \%$ within $72 \mathrm{~h}$ & 13 & 52 \\
& & & \\
Adequacy of Protein Intake & $\geq 80 \%$ within $72 \mathrm{~h}$ & 12 & 48 \\
& $<80 \%$ within $72 \mathrm{~h}$ & 13 & 52 \\
& & & \\
Gastric Residual Volume & LARGER & 15 & 60 \\
(GRV) & LOWER & 7 & 28 \\
& EQUAL & 3 & 12 \\
\hline
\end{tabular}

Caloric and protein requirements were calculated individually from pocket formula 
The values of GRV before and after the increase in the infusion rate, and the volume difference of the GRV when considering sex, age group (adult and elderly) and medical specialty did not present significant difference. It should be emphasized that none of the evaluated patients had a GRV greater than $350 \mathrm{~mL}$ and there was no pulmonary aspiration during the study period.
A significant negative correlation $(\mathrm{r}=-0.46, \mathrm{p}=0.02)$ was found between the increase in the infusion rate of the diet and the difference in GRV, as illustrated in Figure 1. There was no statistically significant correlation between GRV and age, sex and the diagnosis category of hospitalization.

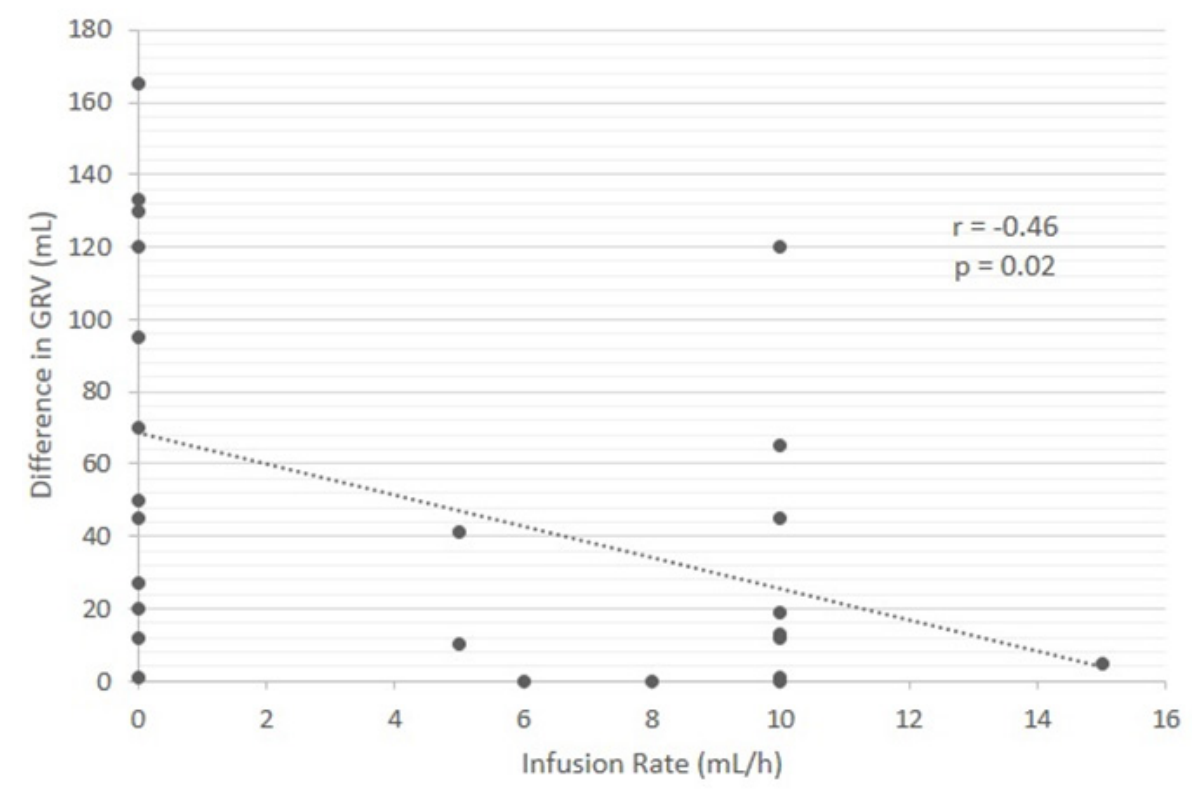

Figure I Correlation between the infusion rate (IR) of the diet and the difference in GRV of patients in ICU.

\section{Discussion}

The development and use of markers that assess gastrointestinal function are constantly under discussion, but no single marker is able to determine food tolerance. These markers include auscultation of bowel sounds, presence of bowel movements, gastric residual volume, abdominal distension and discomfort, nausea, vomiting, and bronchoaspiration. The lack of correlation between these markers, gastrointestinal function, and the risks preceded by intolerance are several times causes of delays and interruption of enteral nutrition unnecessarily. ${ }^{6,11,12}$ In the present study, GRV was evaluated as a marker of EN tolerance, that is, it was evaluated whether there was an association between the increase in the infusion rate of the EN and the GRV found in critically ill patients.

From the results found in this study, it is observed that the GRV before the increase of the infusion rate of the EN was close to that obtained after the elevation of the infusion rate. However, it is known that a marked increase in dietary infusion rate in critically ill patients, who tend to present alterations in gastric motility, may contribute to an increase in GRV. ${ }^{11}$

Despite the poor association between GRV and gastric emptying, this market is still commonly used in patients undergoing EN. Attention to delayed gastric emptying is related to the risk of aspiration and consequently to the development of pneumonia. In this study, the occurrence of bronchoaspiration in the evaluated patients was not evidenced. It is probable that the low GRV observed is one of the factors that contributed to the non-occurrence of this complication.

Other studies that evaluated these variables showed no correlation between the GRV and the risk of bronchoaspiration. Factors such as head-of-bed elevation, feeding tube positioning, Glasgow Scale and gastroesophageal reflux disease were associated with such complication. ${ }^{12,13}$ Although the last two factors were not evaluated in this study, the control of head elevation and positioning of the tube were monitored routinely, also contributing to the non-occurrence of pulmonary aspiration in these patients.

In the unit where the study was carried out, the value proposed as a threshold for maintenance of enteral nutrition was $350 \mathrm{~mL}$, but other measures were also adopted in the nutritional monitoring of the patients, which seemed to contribute to the non-suspension of EN of the patients during the evaluation period. Considering the goal of nutritional adequacy adopted at the hospital and that at least $80 \%$ of the protein-calorie recommendations were achieved within 72 hours, it was evidenced that the nutritional adequacy of the patients evaluated was low. The majority $(52 \%)$ did not reach their nutritional needs within 3days after the beginning of the EN. A delay in the evolution of the diet was observed, since a great part managed to reach its needs in the four days of EN therapy. It is very likely that the increase in GRV, observed in $60 \%$ of the patients, contributed in conjunction with other factors, in the bigger period to reach nutritional needs.

It is known that, in critical patients, gastrointestinal function may be a challenge in the success of EN. ${ }^{11,14}$ A gastrointestinal complication can lead to delayed gastric emptying. Studies indicate a relationship between the presence of food intolerance and reduced nutritional adequacy, longer mechanical ventilation and ICU stay. Patients with sepsis, cardiovascular and gastrointestinal tract diseases are more likely to have these complications, ${ }^{15-18}$ which was not observed in the present study. Negative correlation was evidenced between the increase in infusion rate of the diet and the difference in the GRV, 
that is, the increase in the infusion rate of the diet did not increase the GRV. Our result reinforces that this should not be a marker used alone in order to delay the progression of EN. The elevation of the infusion rate, with greater flow of nutrients to the stomach, is a stimulus for gastric motility. Neurohumoral reflexes involved in the regulation of gastric secretion also affect their motility. The gastric distension leads to secretion of gastrin, which, in turn, enhances the force of contractions and increases the chyme's mixture, which leads to gastric emptying. This factor may explain the lower GRV when the infusion rate increased. ${ }^{19,20}$

Our study presents some limitations. Primarily, the sample size was small. Furthermore, since ours was a retrospective study, the data were dependent on available records, resulting in incomplete assessment of the others variables related. Nevertheless, we performed a general evaluation of the association of GRV and progression of EN, allowing a critical analysis of the use of this indicator.

Therefore, monitoring of gastrointestinal function is essential and must be performed regularly with the aim of: To detect any complication early and to initiate treatment; To reduce and/or prevent pulmonary complications; To optimize the management of EN. ${ }^{8,14,21}$

Considering the findings, the evaluation of GRV is a non-standard practice, which alone does not correlate with gastrointestinal complication, in addition to causing resource and time allocation. However, the choice of use of this indicator should be defined considering the individual aspects of the patients and the other markers of gastrointestinal tolerance, as well as the protocols and practice of the service.

\section{Conclusion}

The results indicate that the increase in the infusion rate of the diet did not contribute to the increase in the GRV of critical patients as the latter is dependent of other signs of intolerance in order to determine gastric emptying. The joint action of the team and the implementation of measures aimed at minimizing the complications inherent to the clinical situation of the patient, providing an adequate nutritional supply, should be applied. It is important for practitioners to be aware of the limitations of GRV assessment and to consider its use individually.

\section{Acknowledgments}

The authors would like to acknowledge the involvement of each study participant and the contribution of the entire team of the University Hospital of the Federal University of Juiz de Fora.

\section{Conflicts of interest}

The authors declare that they have no conflicts of interest to disclose.

\section{Financial disclosure}

None.

\section{Ethical considerations}

This study was conducted according to the principles of the 2013 revised World Medical Association's Declaration of Helsinki and the Medical Research Involving Human Subjects Act. This study was approved by the Committee of Ethics in Research on Human Beings of the University Hospital of the Federal University of Juiz de Fora (No. 2.458.173).

\section{References}

1. Heydari A, Zeydi AE. Is gastric residual volume monitoring in critically ill patients receiving mechanical ventilation an evidence-based practice? Indian J Crit Care Med. 2014;18:259260.

2. Reignier J, Mercier E, Le Gouge A, et al. Effect of Not Monitoring Residual Gastric Volume on Risk of Ventilator-Associated Pneumonia in Adults Receiving Mechanical Ventilation and Early Enteral Feeding. JAMA. 2013;309:249-256.

3. Mentec H, Dupont H, Bocchetti M, et al. Upper digestive intolerance during enteral nutrition in critically ill patients. Crit Care Med. 2001;29(10):1955-1961.

4. Chang WK, McClave SA, Lee MS, et al. Monitoring bolus nasogastric tube feeding by the brix value determination and residual volume measurement of gastric contents. JPEN. 2003;124(4):429.

5. McClave SA, Snider HL, Lowen CC, et al. Use of residual volume as a marker for enteral feeding intolerance. JPEN J Parenter Enteral Nutr. 1992;16;99-105.

6. Deane A, Chapman MJ, Fraser RJ, et al. Mechanisms underlying feed intolerance in the critically ill: implications for treatment. World J Gastroenterol. 2007;13:3909-3917.

7. McClave SA, Taylor BE, Martindale RG, et al. Guidelines for the Provision and Assessment of Nutrition Support Therapy in the Adult Critically Ill Patient: Society of Critical Care Medicine (SCCM) and American Society for Parenteral and Enteral Nutrition (A.S.P.E.N.). JPEN J Parenter Enteral Nutr. 2016;40(2):159-211.

8. Bankhead R, Boullata J, Brantley S, et al. A.S.P.E.N. Board of Directors. Enteral nutrition practice recommendations. JPEN $J$ Parenter Enteral Nutr. 2009;33:122-167.

9. Btaiche IF, Chan LN, Pleva M, et al. Critical Illness, Gastrointestinal Complications, and Medication Therapy during Enteral Feeding in Critically Ill Adult Patients. Nutr Clin Pract. 2010;25(1):32-49.

10. McClave SA, Lukan JK, Stefater JA, et al. Poor validity of residual volumes as a marker for risk of aspiration in critically ill patients. Crit Care Med. 2005;33(2):324-330.

11. McClave SA, Martindale RG, Vanek VW, et al. A.S.P.E.N. Board of Directors; American College of Critical Care Medicine;Society of Critical Care Medicine. Guidelines for the Provision and Assessment of Nutrition Support Therapy in the Adult Critically Ill Patient: Society of Critical Care Medicine (SCCM) and American Society for Parenteral and Enteral Nutrition (A.S.P.E.N.). JPEN J Parenter Enteral Nutr. 2009;3(2):277-316.

12. Mallampalli A, McClave SA, Snider HL. Defining tolerance to enteral feeding in the intensive care unit. Clin Nutr. 2000;19(4):213-215. 
13. Metheny NA, Clouse RE, Chang YH, et al. Tracheobronchial aspiration of gastric contents in critically ill tube-fed patients: frequency, outcomes, and risk factors. Crit Care Med. 2006;34:1007-1015.

14. McClave SA, Heyland DK. The physiologic response and associated clinical benefits from provision of early enteral nutrition. Nutr Clin Pract. 2009;24(3):305-315.

15. Gungabissoon U, Hacquoil K, Bains C, et al. Prevalence, risk factors, clinical consequences, and treatment of enteral feed intolerance during critical illness. JPEN J Parenter Enteral Nutr. 2015;39(4):441-448.

16. Nguyen NQ, Ng MP, Chapman M, et al. The impact of admission diagnosis on gastric emptying in critically ill patients. Crit Care. 2007;11:R16.

17. Ukleja A. Altered GI motility in critically Ill patients: current understanding of pathophysiology, clinical impact, and diagnostic approach. Nutr Clin Pract. 2010;25(1):16-25.
18. Elke G, Felbinger TW, Heyland DK. Gastric Residual Volume in Critically Ill Patients: A Dead Marker or Still Alive? Nutr Clin Pract. 2015;30(1):59-71.

19. Browning KN, Travagli RA. Central nervous system control of gastrointestinal motility and secretion and modulation of gastrointestinal functions. Comprehensive Physiology. 2014;4:13339.

20. Camilleri M. Integrated upper gastrointestinal response to food intake. Gastroenterology. 2006;131(2):640.

21. Hsu CW, Sun SF, Lee DL, et al. Impact of disease severity on gastric residual volume in critical patients. World J Gastroenterol. 2011;17:2007-2012. 